\title{
Modeling PSInSAR Time Series Without Phase Unwrapping
}

\author{
Lei Zhang, Student Member, IEEE, Xiaoli Ding, and Zhong Lu, Senior Member, IEEE
}

\begin{abstract}
In this paper, we propose a least-squares-based method for multitemporal synthetic aperture radar interferometry that allows one to estimate deformations without the need of phase unwrapping. The method utilizes a series of multimaster wrapped differential interferograms with short baselines and focuses on arcs at which there are no phase ambiguities. An outlier detector is used to identify and remove the arcs with phase ambiguities, and a pseudoinverse of the variance-covariance matrix is used as the weight matrix of the correlated observations. The deformation rates at coherent points are estimated with a least squares model constrained by reference points. The proposed approach is verified with a set of simulated data.
\end{abstract}

Index Terms-Interferometric synthetic aperture radar (InSAR), least squares, phase ambiguity, phase unwrapping, synthetic aperture radar (SAR).

\section{INTRODUCTION}

$\mathbf{S}$ PACEBORNE interferometric synthetic aperture radar (InSAR) is a valuable technique for measuring surface deformations due to its high spatial resolution and its ability of acquiring data remotely. However, the changing scattering properties of the Earth's surface with time and/or radar look direction often limit the applicability of InSAR [1]. Ground displacement signals are usually mixed with noise due to variations in the atmospheric conditions and errors such as those in satellite orbits and surface elevation models.

The emergence of techniques for analyzing multitemporal SAR images has enhanced the ability of deformation mapping with InSAR [2]. Multitemporal InSAR techniques, involving the processing of multiple-temporal InSAR images, provide a means to address issues in conventional InSAR techniques, such as decorrelation and atmospheric artifacts. Over recent years, a multitude of approaches has been proposed in this domain, which can be broadly classified into two categories, namely, permanent scatterer [(PS); or persistent scatterer as

Manuscript received December 9, 2009; revised May 10, 2010; accepted May 23, 2010. Date of publication July 23, 2010; date of current version December 27, 2010. This work was supported in part by the Research Grants Council of the Hong Kong Special Administrative Region under Project PolyU 5155/07E, by the Faculty of Construction and Land Use of The Hong Kong Polytechnic University under the Sustainable Urbanization Research Fund, by the NASA's Earth Surface and Interiors Program 2005-0021, and by the U.S. Geological Survey Land Remote Sensing and Volcano Hazards Programs. The work of L. Zhang was supported by a Ph.D. studentship offered by The Hong Kong Polytechnic University.

L. Zhang and X. Ding are with The Hong Kong Polytechnic University, Kowloon, Hong Kong (e-mail: 1.zhang@polyu.edu.hk; lsxlding@ polyu. edu.hk).

$\mathrm{Z}$. Lu is with the Earth Resources Observation and Science Center and the Cascades Volcano Observatory, U.S. Geological Survey, Vancouver, WA 98683 USA (e-mail: lu@usgs.gov).

Color versions of one or more of the figures in this paper are available online at http://ieeexplore.ieee.org.

Digital Object Identifier 10.1109/TGRS.2010.2052625 used in some literature] [3], [4], [7], [8], [16], [26] and small baseline subset (SBAS) InSAR methods [9]-[12], [17]. One common drawback of both PSInSAR and SBAS methods is the requirement of phase unwrapping. Because of errors induced from phase unwrapping, both techniques sometimes fail to estimate correctly the parameters [deformation, digital elevation model (DEM) error, and atmospheric delay] from a stack of interferograms.

How to reduce or avoid the unwrapping errors is therefore a challenge that all multitemporal InSAR methods need to overcome. In applying such techniques, we have found that, for a set of multimaster interferograms with short baselines, there are usually a sufficient number of arcs in which the doubledifference phase components due to topographic errors and atmospheric artifacts are very small and the relative deformation rate between two connected points is low. These arcs can be safely considered to be immune from phase ambiguities. If only these arcs are taken as observations for estimating the DEM errors and deformations, the complexity of parameter estimation can be reduced significantly since there is no longer a need of phase unwrapping. In this paper, we propose a least-squaresbased method that can select efficiently arcs without phase ambiguities and resolve reliably the deformation parameters (linear or nonlinear) at coherent points. We first use a network construction strategy that performs Delaunay triangulation locally to ensure that coherent points can be connected as much as possible while not significantly increasing the computational complexity. A least-squares-based model is then proposed for parameter estimation during which an outlier detector is used to identify and remove arcs with phase ambiguities according to the least squares residuals. Considering the stochastic nature of SAR observations, we introduce a weighting scheme for the interferometric phases at arcs by applying the law of variance propagation [13]-[15]. The parameters at the coherent points are then estimated by applying a least squares model constrained by reference points. The proposed approach is tested with a set of simulated SAR data to ensure that the proposed method functions as expected under controlled circumstances [13].

\section{MOdeling SAR InTERFerograms}

\section{A. Coherent Point Network}

The starting point of the method is the identification of PSs, which can be performed using amplitude dispersion index [18] or other thresholds [7], [19]. The methods work for PSs that appear in all the interferograms or only some of the interferograms. We therefore call these points as temporarily coherent 

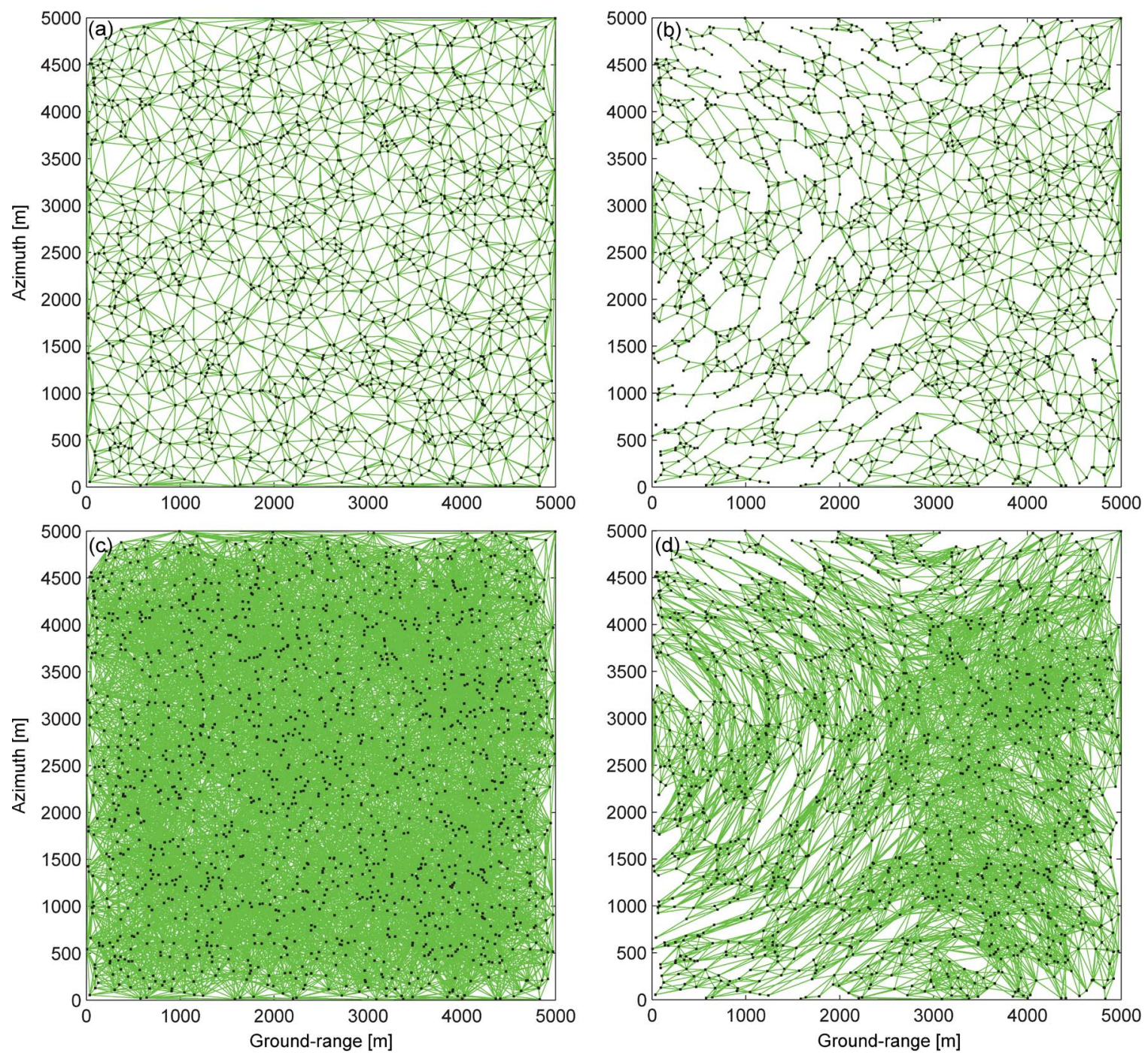

Fig. 1. (a) Global Delaunay triangulation network of coherent points. (b) Network after removing arcs with phase ambiguities detected from (a). (c) Local Delaunay triangulation network of coherent points. (d) Network after removing arcs with phase ambiguities detected from (c).

points (TCPs). Once the TCPs are identified, a network is constructed to connect pairs of TCP, where each connected pair is termed an arc as in PSInSAR terminology. Delaunay triangulation has been widely used for this purpose. However, the Delaunay triangulation defines a triangular network under the condition that the circumcircles of all the triangles in the network are empty without considering the lengths of the arcs [see Fig. 1(a)]. Although arcs longer than a certain length can be removed in the final step, the points are not connected with optimized arc lengths. If only points in a small region (i.e., $1500 \mathrm{~m} \times 1500 \mathrm{~m}$ ) are connected, the problem of arc length can be solved without increasing significantly the computational complexity. Fig. 1(c) shows a local Delaunay triangulation where a grid with $100-\mathrm{m}$ spacing is placed over an interferogram and points in a circle with a radius of $750 \mathrm{~m}$ centered at each grid node are selected and connected.

\section{B. Multiple Master Interferogram Stacking}

Considering $J+1$ SAR images acquired in an ordered time sequence $\left(\begin{array}{llll}t_{0} & t_{1} & \cdots & t_{J}\end{array}\right)$, we generate $I$ interferograms with short baselines (for example, less than $150 \mathrm{~m}$ ). In each interferogram $i$, the line-of-sight (LOS) displacement of TCP $(l, m)$ can be described by a linear combination of the mean deformation rate $\left(\nu_{k}, k=1,2, \ldots, J\right)$ between the acquisitions and the corresponding time span. Given two acquisitions, one is the master (M) image and the other is the slave (S) image, and $\mathbf{M}$ is acquired later than $\mathbf{S}$, i.e., $t_{M_{i}}>t_{S_{i}}$. The LOS deformation $\left(\Delta r_{l, m}^{i}\right)$ during this time period can be expressed as

$$
\Delta r_{l, m}^{i}=r\left(t_{M_{i}}, l, m\right)-r\left(t_{S_{i}}, l, m\right)=\sum_{k=1}^{C_{i}-1}\left(t_{k}-t_{k-1}\right) \nu_{k}
$$

where $(l, m)$ are the pixel coordinate of the TCP; $r\left(t_{M_{i}}, l, m\right)$ and $r\left(t_{S_{i}}, l, m\right)$ are the slant range distances from the master and slave sensors, respectively, to the target; and $C_{i}$ is the number of single-look complex (SLC) acquisitions in the time sequence from $S_{i}$ to $M_{i}$ (including $M_{i}$ and $S_{i}$ ).

As (1) is a combination of LOS deformation estimates at the highest time resolution, there is a risk of overparameterization 
in the equation, which should be carefully dealt with in a real application. If a linear deformation rate $(\nu)$ during the whole time span is assumed, then $\nu_{1}=\cdots=\nu_{k}=\cdots=\nu$. Equation (1) can also be tailored as any combination of deformation rates and time intervals in order to compare with field measurements that, for example, are performed annually. The corresponding phase is

$$
\begin{aligned}
\phi_{\text {defo }, l, m}^{i} & =-\frac{4 \pi}{\lambda} \Delta r_{l, m}^{i}=-\frac{4 \pi}{\lambda} \sum_{k=1}^{C_{i}-1}\left(t_{k}-t_{k-1}\right) \nu_{k} \\
& =\beta_{i} V
\end{aligned}
$$

where $\lambda$ is the radar wavelength, $V$ is a vector of deformation rates, $\beta_{i}=-(4 \pi / \lambda) T_{i}$, and $T_{i}$ is a vector of time combinations whose elements correspond to the deformation rates within the respective time intervals

$$
T_{i}=\left[\begin{array}{llllll}
t_{1}-t_{0} & t_{2}-t_{1} & \cdots & t_{k}-t_{k-1} & \cdots & t_{J}-t_{J-1}
\end{array}\right]_{1 \times J} .
$$

The wrapped phase of a TCP with a pixel coordinate of $(l, m)$ can be written as

$$
\begin{aligned}
\phi_{l, m}^{i}=\phi_{\mathrm{topo}, l, m}^{i}+ & \phi_{\mathrm{defo}, l, m}^{i}+\phi_{\mathrm{atmo}, l, m}^{i} \\
& +\phi_{\mathrm{orbit}, l, m}^{i}+\phi_{\mathrm{dop}, l, m}^{i}+\phi_{\text {noise }, l, m}^{i}
\end{aligned}
$$

where $\phi_{\mathrm{topo}, l, m}^{i}$ is the phase related to the topographic error, $\phi_{\mathrm{atmo}, l, m}^{i}$ is the phase due to the differential atmospheric delays between the acquisitions, $\phi_{\text {orbit }, l, m}^{i}$ is the phase due to the orbit errors, $\phi_{\mathrm{dop}, l, m}^{i}$ is the phase component due to azimuth Doppler centroid difference between the acquisitions, and $\phi_{\text {noise }, l, m}^{i}$ is the noise term that includes potentially the thermal noise, processing errors, and decorrelation errors. The $\phi_{\text {topo }, l, m}^{i}$ term has a direct relationship with the height error $\Delta h_{l, m}$

$$
\begin{aligned}
\phi_{\text {topo }, l, m}^{i} & =-\frac{4 \pi}{\lambda} \frac{B_{\perp, l, m}^{i}}{r_{l, m}^{i} \sin \theta_{l, m}^{i}} \Delta h_{l, m} \\
& =\alpha_{l, m}^{i} \Delta h_{l, m}
\end{aligned}
$$

where $B_{\perp, l, m}^{i}$ is the local perpendicular baseline, $r_{l, m}^{i}$ is the slant range distance from the master sensor to the target, and $\theta_{l, m}^{i}$ is the local incident angle.

The phase difference between two TCPs located at $(l, m)$ and $\left(l^{\prime}, m^{\prime}\right)$ is given by

$$
\begin{aligned}
\Delta \phi_{l, m, l^{\prime}, m^{\prime}}^{i}= & \alpha_{l, m}^{i} \Delta h_{l, m, l^{\prime}, m^{\prime}}+\beta_{i} \Delta V+w_{l, m, l^{\prime}, m^{\prime}}^{i} \\
w_{l, m, l^{\prime}, m^{\prime}}^{i}= & \Delta \phi_{\text {atmo }, l, m, l^{\prime}, m^{\prime}}^{i}+\Delta \phi_{\text {orbit }, l, m, l^{\prime}, m^{\prime}}^{i} \\
& +\Delta \phi_{\mathrm{dop}, l, m, l^{\prime}, m^{\prime}}^{i}+\Delta \phi_{\text {noise }, l, m, l^{\prime} m^{\prime}}^{i}
\end{aligned}
$$

where $\Delta V=\left[\begin{array}{llll}\Delta \nu_{l, m, l^{\prime}, m^{\prime}}^{1} & \Delta \nu_{l, m, l^{\prime}, m^{\prime}}^{2} & \cdots & \Delta \nu_{l, m, l^{\prime}, m^{\prime}}^{I}\end{array}\right]^{\mathrm{T}}$. Since the atmospheric artifacts are strongly correlated in space, the differential atmospheric contributions between a pair of nearby TCPs should be very low (for points that are less than $1 \mathrm{~km}$ apart, $\sigma_{\Delta \phi_{\text {atmo }}}$ is usually lower than $0.1 \operatorname{rad}^{2}$ [23]-[25]). The differential orbital component generally has a similar characteristic. Since the differencing operation can also significantly reduce the effects of Doppler centroid differences,
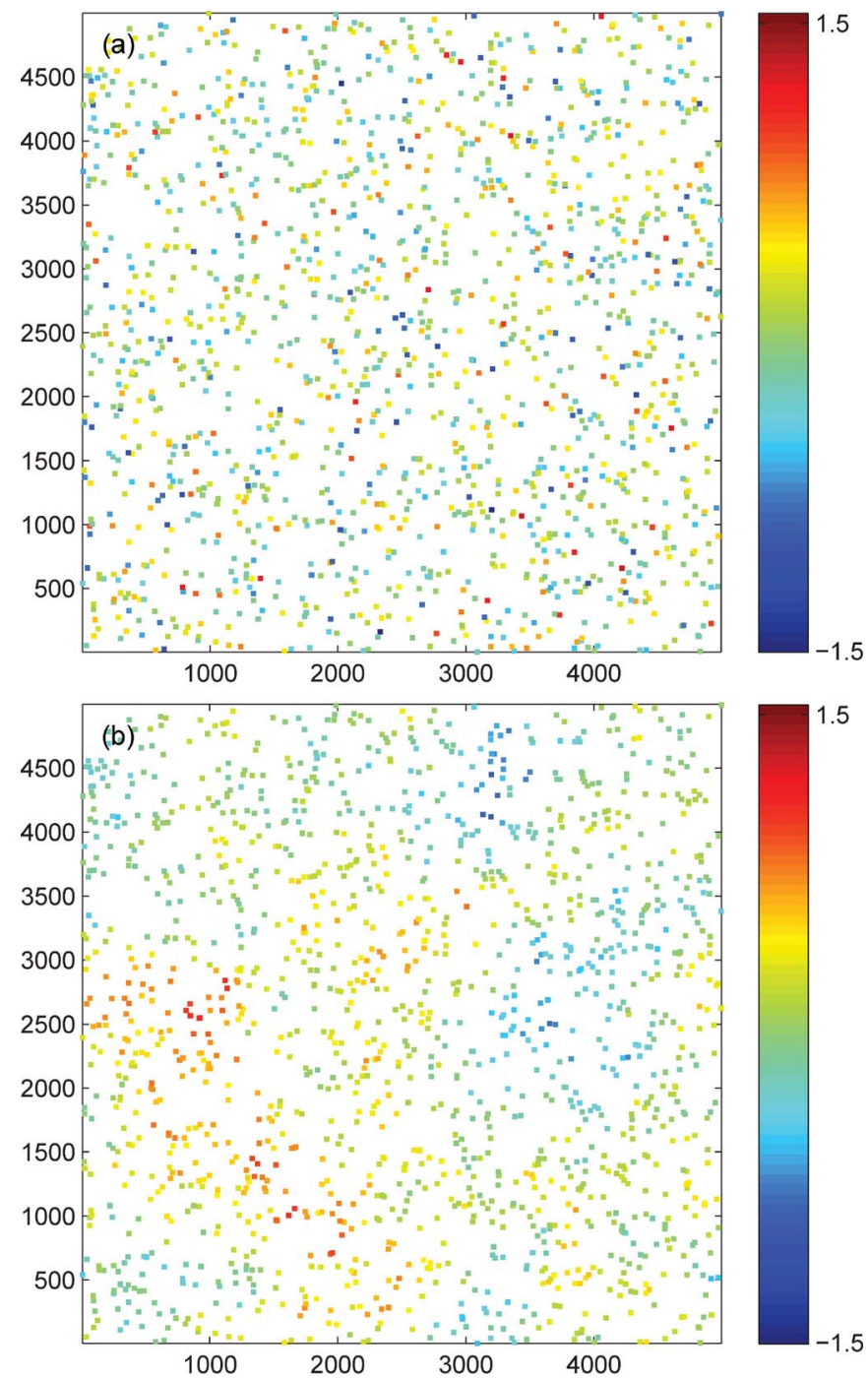

Fig. 2. Examples of simulated signals. (a) Simulated noise in an SLC image. (b) Simulated atmospheric artifact. The unit is rad.

the magnitude of $\Delta \phi_{\mathrm{dop}, l, m, l^{\prime}, m^{\prime}}^{i}$ should be very small. Moreover, if none of the two connected TCPs is significantly affected by decorrelation, $\Delta \phi_{\text {noise }, l, m, l^{\prime} m^{\prime}}^{i}$ will also show a low variance. Therefore, $w_{l, m, l^{\prime}, m^{\prime}}^{i}$ can be safely taken as a random variable with an expectation $E\left(w_{l, m, l^{\prime}, m^{\prime}}^{i}\right)=0$. For arcs without phase ambiguities, the system of observations can be written as

$$
\begin{aligned}
\Delta \Phi & =A\left[\begin{array}{c}
\Delta h_{l, m, l^{\prime}, m^{\prime}} \\
\Delta V
\end{array}\right]+W \\
\Delta \Phi & =\left[\begin{array}{llll}
\Delta \phi_{l, m, l^{\prime}, m^{\prime}}^{1} & \Delta \phi_{l, m, l^{\prime}, m^{\prime}}^{2} & \cdots & \Delta \phi_{l, m, l^{\prime}, m^{\prime}}^{I}
\end{array}\right] \\
A & =\left[\begin{array}{llll}
\alpha & \beta
\end{array}\right] \\
\alpha & =\left[\begin{array}{llll}
\alpha_{l, m}^{1} & \alpha_{l, m}^{2} & \cdots & \alpha_{l, m}^{I}
\end{array}\right]^{T} \\
\beta & =\left[\begin{array}{lllll}
\beta_{1} & \beta_{2} & \cdots & \beta_{I}
\end{array}\right]^{T} \\
W & =\left[\begin{array}{llll}
w_{l, m, l^{\prime}, m^{\prime}}^{1} & w_{l, m, l^{\prime}, m^{\prime}}^{2} & \cdots & w_{l, m, l^{\prime}, m^{\prime}}^{I}
\end{array}\right]
\end{aligned}
$$

where $\Delta \Phi$ is a vector containing phase differences between two adjacent pixels in a total of $I$ interferograms. $A$ is the design matrix including height-to-phase conversion factors and time combination matrix. $W$ is a stochastic vector. 

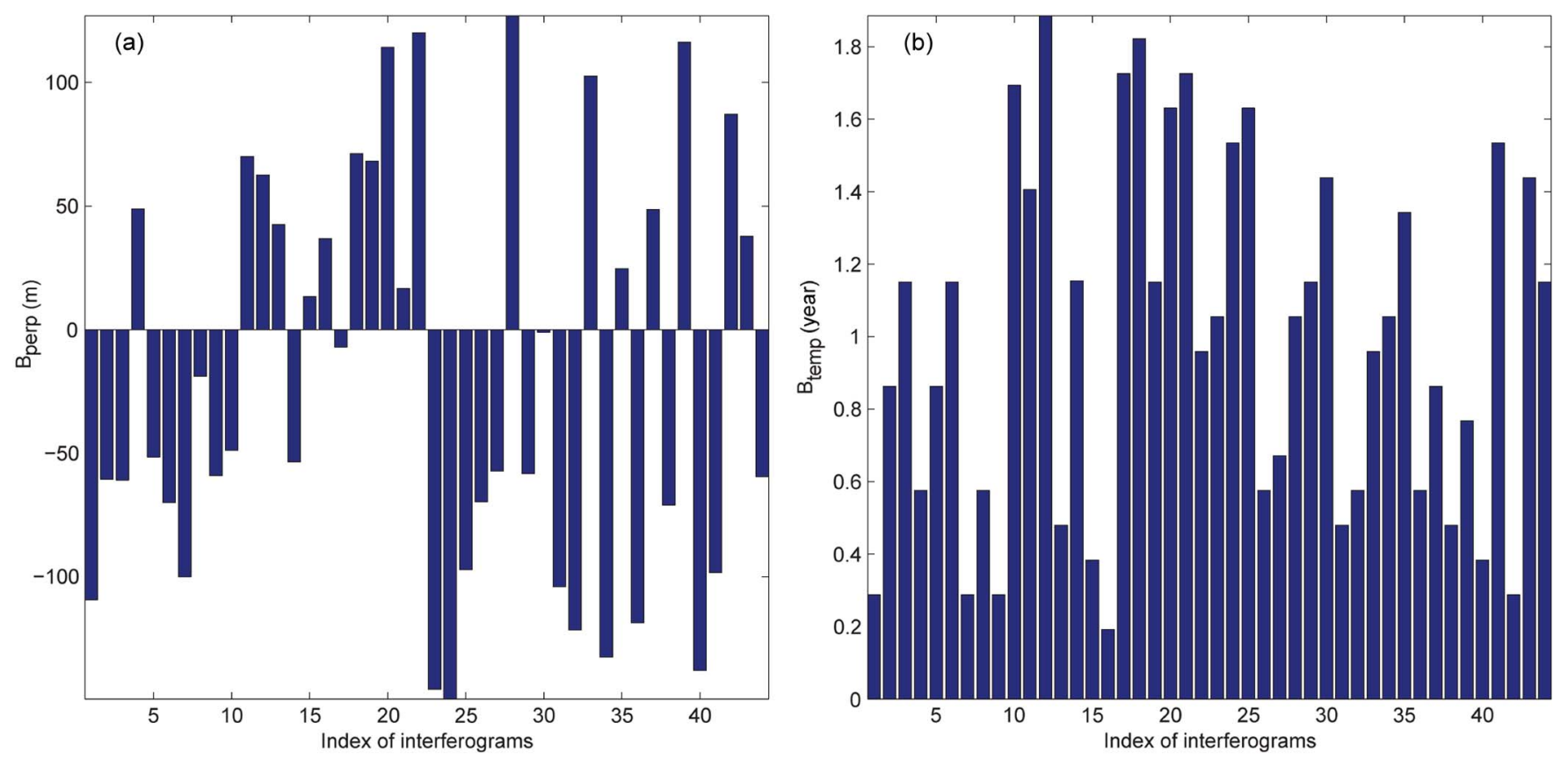

Fig. 3. (a) Perpendicular baselines. (b) Temporal baselines of the 44 simulated interferograms.

\section{Least SQuares Solution}

The functional and the stochastic models of the system of observations can be expressed as

$$
\begin{aligned}
& E\{\Delta \Phi\}=A\left[\begin{array}{c}
\Delta \hat{h}_{l, m, l^{\prime}, m^{\prime}} \\
\Delta V
\end{array}\right]=\Delta \Phi-W \\
& D\{\Delta \Phi\}=Q^{\mathrm{dd}}
\end{aligned}
$$

where $(E\{\cdot\})$ and $(D\{\cdot\})$ are operators for expectation and dispersion, respectively, and $Q^{\mathrm{dd}}$ is an $I \times I$ covariance matrix of observations. The functional model reflects the linear or linearized relationship between the observations and the unknown parameters, while the stochastic model describes the accuracy of the observations and the correlation between them. In this section, a weighted least squares estimator is used to resolve the parameters in (7) and (8).

\section{A. A Priori Variance Components}

In the conventional multitemporal InSAR analysis technique, all double-difference observations are assumed to have equal weights. This assumption may not be valid since SAR images are acquired under different conditions with which the atmospheric artifacts and random noises vary. For the $J+1$ SAR images considered, the variance-covariance (VC) matrix of the random noises $\left(Q_{\text {noise }}^{i}\right)$ of the $i$ th TCP can be expressed as

$$
Q_{\text {noise }}^{i}=\left[\begin{array}{ccc}
\sigma_{\text {noise }_{0}^{i}}^{2} & & \\
& \ddots & \\
& & \sigma_{\text {noise }_{J}^{i}}^{2}
\end{array}\right]
$$

where it is assumed that the noise is uncorrelated among the SAR images. When $I$ interferograms are formed from the $J+1$ images, the VC matrix of the interferogams is given according to the law of variance propagation

$$
Q_{\text {noise }}^{\text {in }}=D Q_{\text {noise }} D^{\mathrm{T}}
$$

where $D$ is a combination matrix indicating which pair of SLC images are used to generate the interferograms. The combination matrix has the following form:

$$
D=\left[\begin{array}{ccccc}
-1 & 0 & 1 & \cdots & 0 \\
0 & -1 & \cdots & 1 & 0 \\
\vdots & \ddots & & & \\
0 & 0 & -1 & \cdots & 1
\end{array}\right]_{I \times(J+1)}
$$

We assume that the all the TCPs in all the SLC images have the variances, i.e., $Q_{\text {noise }}^{1}=Q_{\text {noise }}^{2}=\cdots Q_{\text {noise }}^{H}$, where $H$ is the number of the TCPs. The VC matrix of the doubledifference phases then becomes

$$
Q^{\mathrm{dd}}=2 Q_{\text {noise }}^{\text {in }}=2 D Q_{\text {noise }} D^{\mathrm{T}} .
$$

The atmospheric artifacts in the double-difference phases are treated as stochastic variables behaving like noise in the time domain.

The weights $\left(P^{\mathrm{dd}}\right)$ of the double-difference phases can be obtained by taking the inverse of the VC matrix. Since it is possible that the VC matrix is singular, a pseudoinverse of the VC matrix, i.e., $P^{\mathrm{dd}}=\left(Q^{\mathrm{dd}}\right)^{+}$, is obtained by singular value decomposition [20] when this happens. 


\section{B. Least Squares Solution}

The least squares solution of the observation equations is [18]

$$
\begin{aligned}
{\left[\begin{array}{c}
\Delta \hat{h}_{l, m, l^{\prime}, m^{\prime}} \\
\Delta \hat{V}
\end{array}\right] } & =\left(A^{\mathrm{T}} P^{\mathrm{dd}} A\right)^{-1} A^{\mathrm{T}} P^{\mathrm{dd}} \Delta \Phi \\
\Delta \hat{\Phi} & =A\left(A^{\mathrm{T}} P^{\mathrm{dd}} A\right)^{-1} A^{\mathrm{T}} P^{\mathrm{dd}} \Delta \Phi \\
r & =\Delta \Phi-A\left(A^{\mathrm{T}} P^{\mathrm{dd}} A\right)^{-1} A^{\mathrm{T}} P^{\mathrm{dd}} \Delta \Phi
\end{aligned}
$$

where the circumflex $\hat{\cdot}$ denotes the estimated quantities, and $r$ is the least squares residuals. The corresponding VC matrices of the estimated quantities are

$$
\begin{aligned}
D\left\{\left[\begin{array}{c}
\Delta \hat{h}_{l, m, l^{\prime}, m^{\prime}} \\
\Delta \hat{V}
\end{array}\right]\right\} & =Q_{\hat{x} \hat{x}}=\left(A^{\mathrm{T}} P^{\mathrm{dd}} A\right)^{-1} \\
D\{\Delta \hat{\Phi}\} & =Q_{\Delta \hat{\Phi} \Delta \hat{\Phi}}=A\left(A^{\mathrm{T}} P^{\mathrm{dd}} A\right)^{-1} A^{\mathrm{T}} \\
D\{r\} & =Q_{\nu \nu}=Q_{\mathrm{dd}}-A\left(A^{\mathrm{T}} P^{\mathrm{dd}} A\right)^{-1} A^{\mathrm{T}} .
\end{aligned}
$$

\section{Outlier Detection}

A basic assumption of any least squares estimation is that all the gross errors and systematic effects have been eliminated before the adjustment computation is performed. However, during the initial least squares estimation, all arcs, including those with phase ambiguities in some interferograms, are used as observations. We detect those arcs with phase ambiguities by applying an outlier detector and remove them from the solution. Methods based on statistical tests of the estimated least squares residuals are often used for this purpose [15]. However, they are inefficient as statistical testing should be carried out for each of the iterative least squares solutions. Because double-difference phases with phase ambiguities $(N \cdot 2 \pi, N=1,2, \ldots, n)$ render the magnitude of the corresponding residuals to increase significantly, and we are just interested in whether the arcs have ambiguities or not and there is no need to detect exactly which interferograms have ambiguities, we use a simplified outlier detector [21]

$$
\operatorname{Max}\left(\left|r_{i}\right|\right)>c \sqrt{\operatorname{Max}\left(\left(Q^{\mathrm{dd}}\right)_{i i}\right)}+2 \sqrt{\operatorname{Max}\left(\left(Q_{\Delta \hat{\Phi} \Delta \hat{\Phi}}\right)_{i i}\right)}
$$

where $\operatorname{Max}(\cdot)$ means the maximum value in a vector or matrix. According to [21], the constant $c$ may be chosen as 3 or 4 . When the threshold value in (15) is reached, the $i$ th observation is considered an outlier at $95 \%$ confidence level.

\section{Final Solution}

After removing arcs with phase ambiguities, a least squares solution is obtained finally to estimate the unknown parameters. Compared with the integer least squares estimator and the method based on maximization of the ensemble coherence, the proposed method determines more efficiently and reliably the DEM errors and the differential deformation rates along the arcs since there is no need to perform a search of phase ambiguities in the solution space. Once the parameters along the arcs are determined, parameters at the points can be obtained by
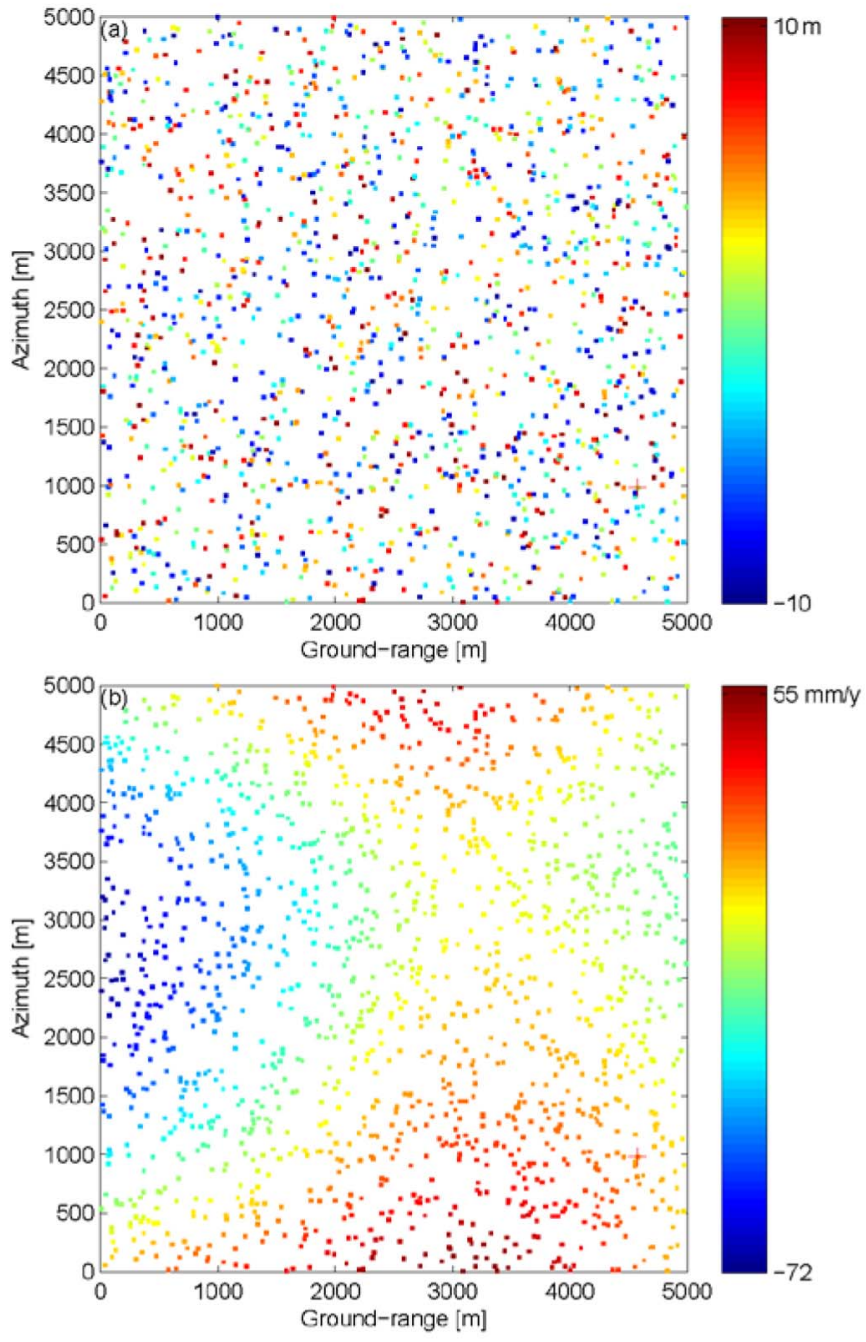

Fig. 4. (a) Simulated DEM errors. (b) Simulated linear deformation rates. The red cross indicates the position of the reference point.

spatial integration, which can also be performed under a least squares framework. The arcs and the points can be linked by a design matrix $U$

$$
L=U X_{o}
$$

where $L$ is the parameters at the arcs, and $X_{o}$ is the parameters at the points

$$
\begin{aligned}
X_{o}^{\prime} & =\left[\begin{array}{llllllll}
x_{1} & x_{2} & \cdots & x_{i-1} & x_{i} & x_{i+1} & \cdots & x_{H}
\end{array}\right] \\
x_{i} & =\left[\begin{array}{llllll}
h_{i} & V_{i}
\end{array}\right] \\
U & =\left[\begin{array}{cccccc}
1 & -1 & 0 & 0 & \cdots & 0 \\
1 & 0 & -1 & 0 & \cdots & 0 \\
\vdots & & & & & \\
0 & 0 & 1 & -1 & \cdots & 0 \\
\vdots & & & & & \\
0 & 0 & 0 & \cdots & 1 & -1
\end{array}\right]_{G \times H}
\end{aligned}
$$

where $G$ is the number of arcs and $H$ is the number of points. $U$ is built according to the records of the starting and the stopping point for each arc during network construction. We set 1 for the 


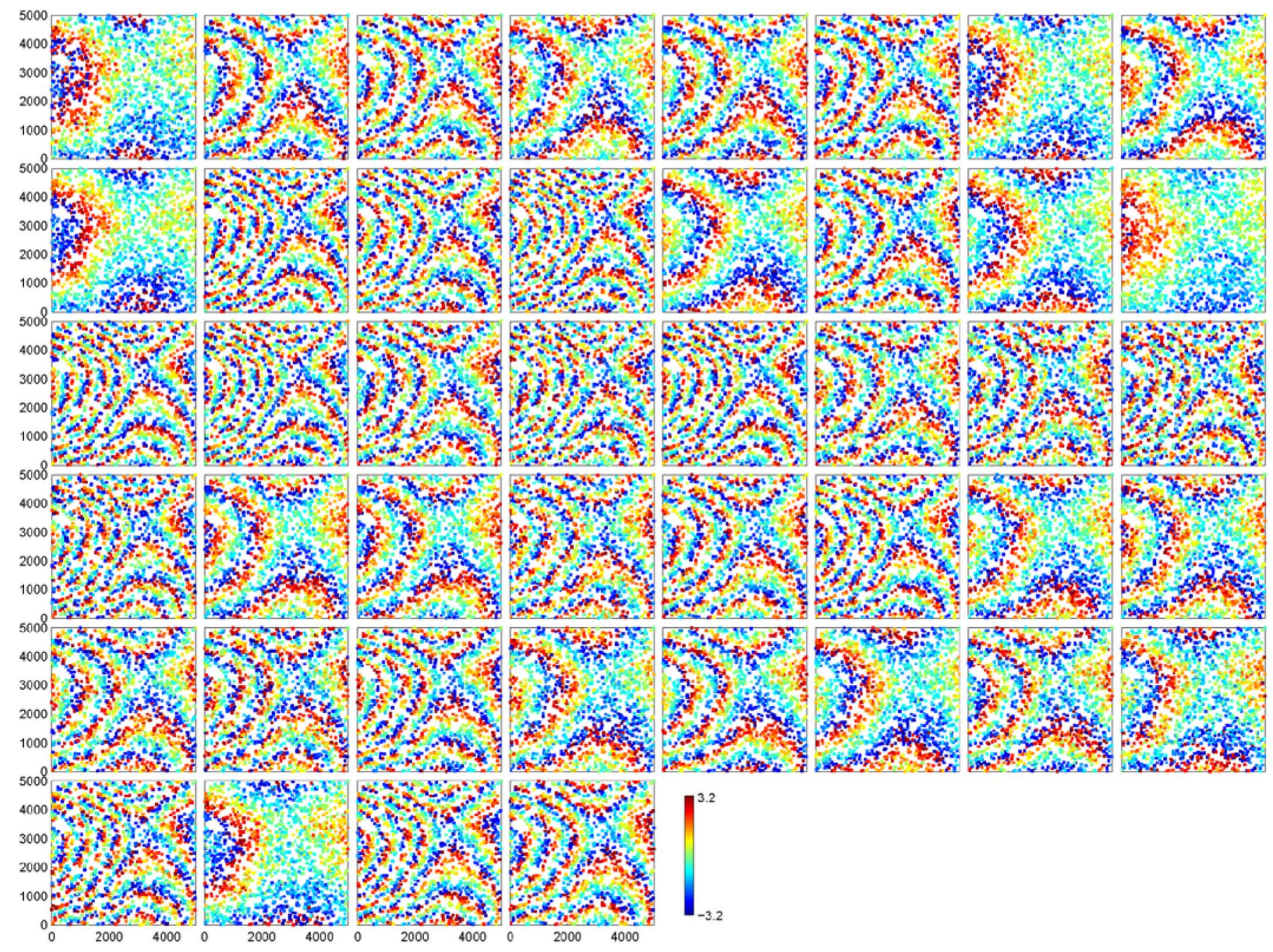

Fig. 5. Simulated wrapped phase at coherent points in the 44 interferograms. Phase values contain components corresponding to DEM errors, deformation, atmospheric artifacts, and random noise. The unit is rad.

starting point, -1 for the stopping point, and 0 if otherwise in the matrix $U$.

The rank of the design matrix $U$ is always one less than the number of TCPs. As a result, the system must be solved relative to a reference point at which the parameters are known. Let the $i$ th point be the reference point with known parameters $\left(R_{i}\right)$, and its corresponding column in $U$ is $S_{i}$. By introducing $L_{L}$ with $L_{L}=L-S_{i} R_{i}$, we obtain

$$
L_{L}=U_{U} X
$$

where $U_{U}$ is an updated design matrix in which the $i$ th column has been removed and $X$ is the parameter matrix at all points except the reference point. The least squares solution is

$$
X=\left(U_{U}^{\mathrm{T}} P U_{U}\right)^{-1} U_{U}^{\mathrm{T}} P L_{L}
$$

where $P=I$. If more than one reference points (for example, $N, N \geq 2$ ) are available, the parameters can be solved by adding constraints

$$
\underset{N-1, H}{C} \underset{H, 1}{X}+\underset{N-1,1}{M}=0
$$

where $C$ is a design matrix indicating the positions of the $(N-1)$ reference points and $M_{x}$ is the known parameters at the $(N-1)$ reference points. The solution is

$$
X=\left(N_{B B}^{-1}-N_{B B}^{-1} C^{\mathrm{T}} N_{C C}^{-1} C N_{B B}^{-1}\right) Z-N_{B B}^{-1} C^{\mathrm{T}} N_{C C}^{-1} M_{x}
$$

where $\quad N_{B B}=U_{U}^{\mathrm{T}} P U_{U}, \quad Z=U_{U}^{\mathrm{T}} P L_{L}, \quad$ and $\quad N_{C C}=$ $C N_{B B}^{-1} C^{\mathrm{T}}$.

\section{Validation With Simulated Data}

A data set consisting of 21 simulated C-band images is used to validate the proposed approach. The advantage of using simulated data is that the estimated parameters can be compared with their true values that are often not known in the case of real data sets [13]. During the simulation, the random phase noise levels in all images are set to a mean of $15^{\circ}$ with a standard deviation of $5^{\circ}$, and the atmospheric phase is simulated using fractal surfaces with a dimension of 2.67. More details about the simulation can be found in [13] and [22]. An example of the simulated noise and atmospheric signal is shown in Fig. 2. Forty-four interferograms with perpendicular 

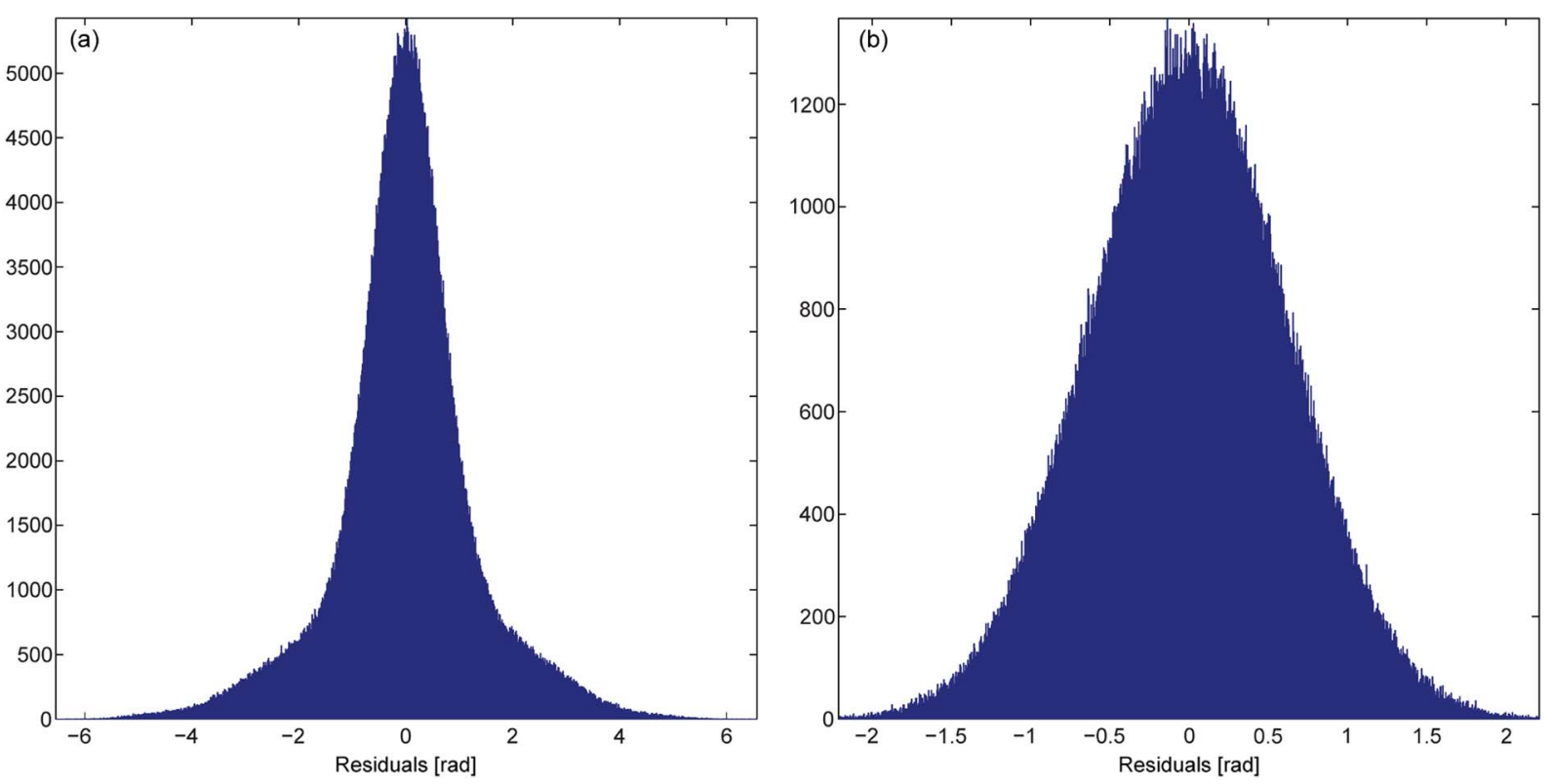

Fig. 6. (a) Histogram of least squares residuals for all observations. (b) Histogram of least squares residuals after removing arcs with ambiguities.
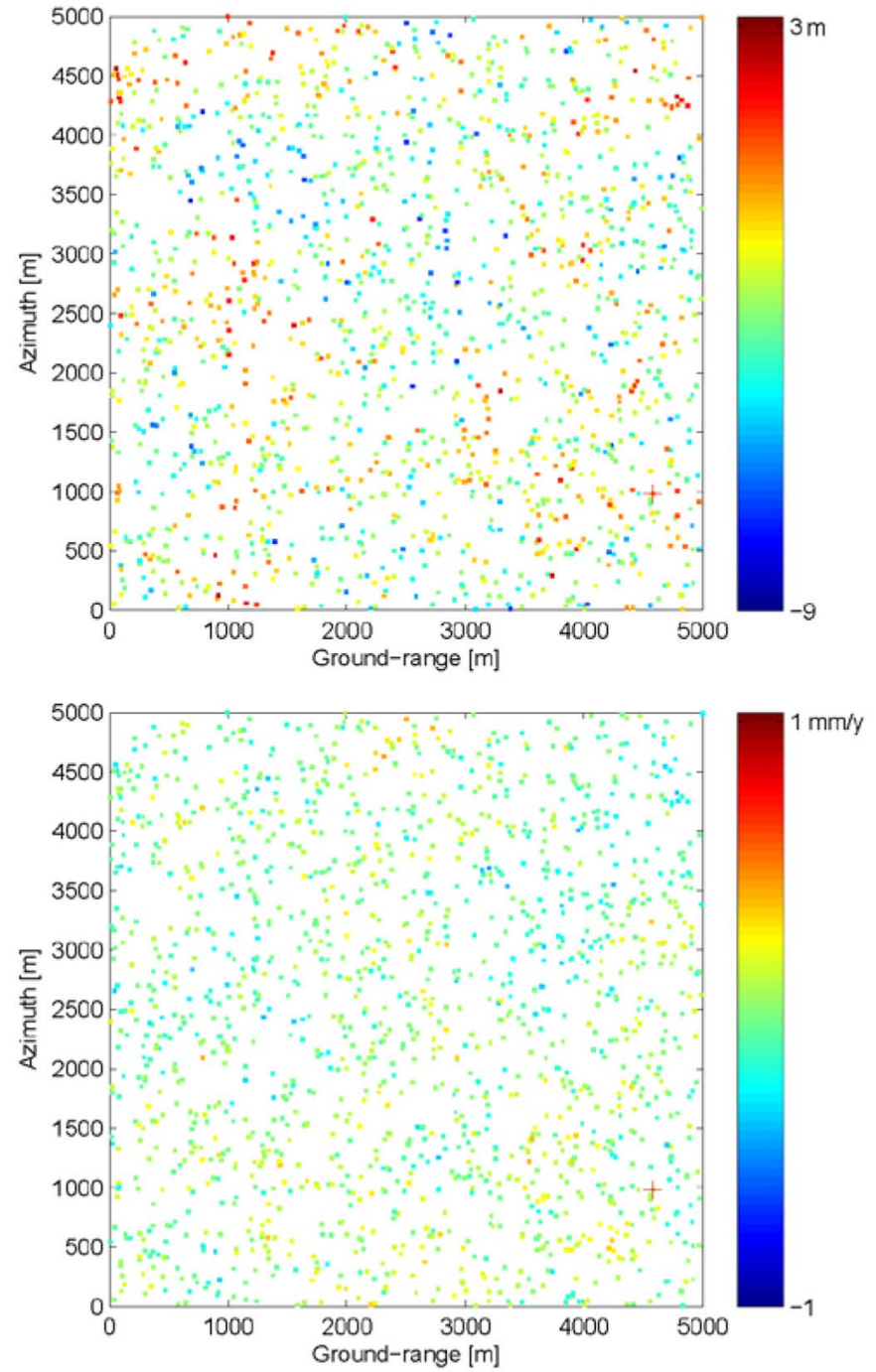

Fig. 7. (a) Errors in the estimated DEM at the TCP. (b) Errors in the estimated deformation rate at the TCP.
TABLE I

STATISTICS OF ERRORS IN THE ESTIMATED PARAMETERS

\begin{tabular}{ccccc}
\hline & $\min$ & $\max$ & mean & Std* $^{*}$ \\
\hline DEM error $(\mathrm{m})$ & -8.1 & 2.4 & -2.6 & $1.72(1.64)$ \\
Linear defo. rate $(\mathrm{mm} / \mathrm{y})$ & -0.45 & 0.41 & -0.008 & $0.164(0.137)$ \\
\hline
\end{tabular}

$(*)$ : Standard deviation from Eq.(14)

and temporal baselines that are shorter than $150 \mathrm{~m}$ and two years, respectively, are produced from the 21 images (Fig. 3). Within an area of $5 \times 5 \mathrm{~km}^{2}, 1500$ TCPs are selected. The simulated DEM errors at the TCP are shown in Fig. 4(a). Both linear and nonlinear deformation models are simulated to test the robustness of the proposed method.

\section{A. Estimation of Linear Deformation Signal}

Linear deformation rates with a maximum magnitude of $72 \mathrm{~mm} /$ year [Fig.4(b)] are first simulated to test the performance of the proposed method. The phase contributions from the DEM errors, deformation, noise, atmospheric artifacts, and orbital inaccuracy are shown in Fig. 5. For testing purposes, we first connect the 1500 TCPs by a global Delaunay triangulation network [Fig. 1(a)]. After removing the arcs with phase ambiguities by the outlier detector, it is found that the network constructed by the remaining arcs is too sparse to estimate the parameters at all the points. We then adopt the local triangulation strategy as described in Section II-A to generate a network of 20091 arcs [Fig. 1(c)]. The longest arc has a distance of $1454 \mathrm{~m}$. A histogram of the absolute residuals from the first least squares estimation is shown in Fig. 6(a). Some 9711 arcs are detected as outliers by applying the outlier detector and removed from the network. The actual number of arcs with phase ambiguities is 9535, all of which have been successfully identified. This also means that 176 arcs have been misidentified. After removing the detected arcs, the 

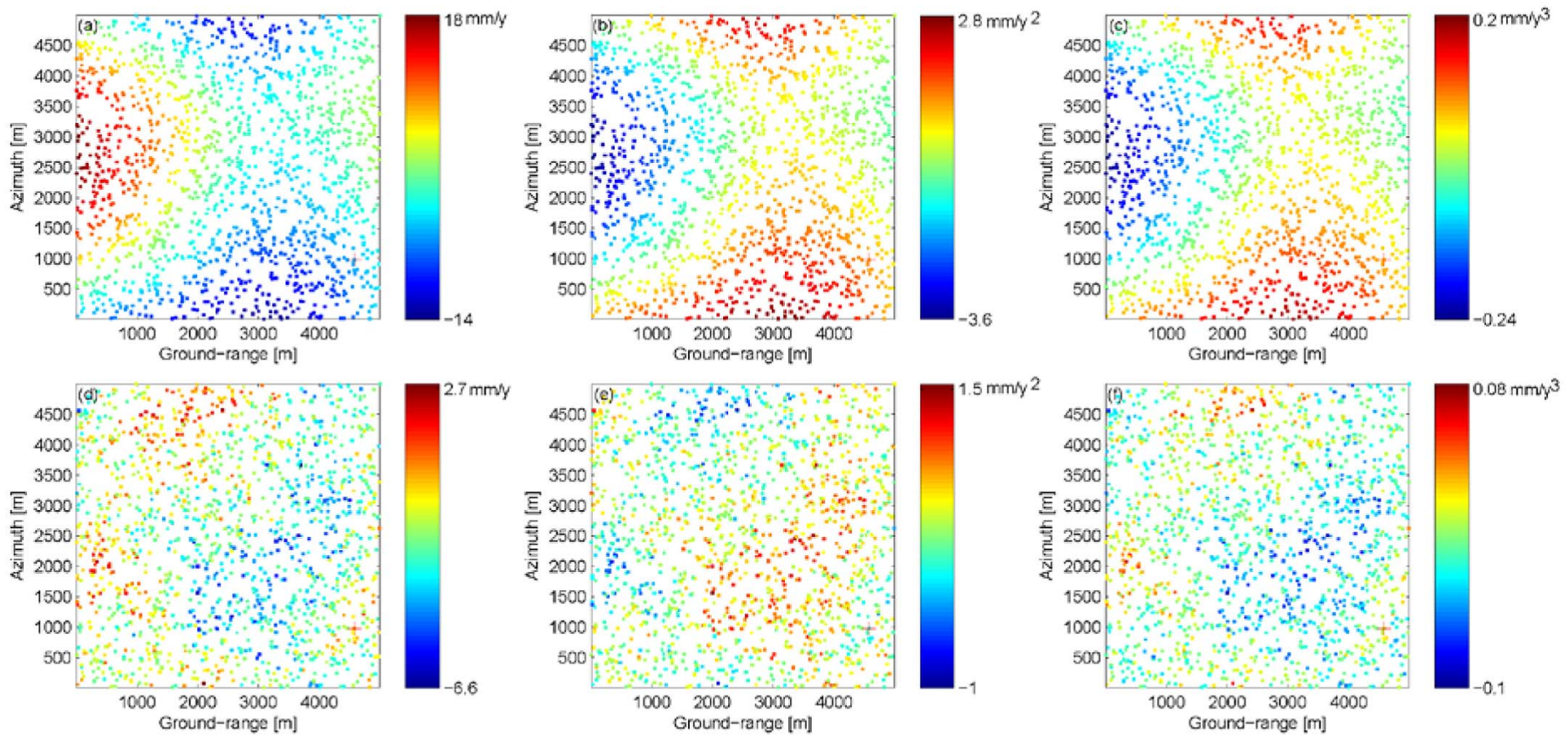

Fig. 8. (a)-(c) Coefficients to be estimated at each TCP, linear term, quadratic term, and cubic term, respectively. (d)-(f) Corresponding errors in the estimated coefficients.

least squares estimator is performed again. Fig. 6(b) shows the updated residuals of the remaining arcs.

Using the a priori $\mathrm{VC}$ matrix for the double-difference phase observations, the $\mathrm{VC}$ matrix of the estimated parameters (the DEM error and the differential deformation rate) at each point is [see (14)]

$$
Q_{\Delta h, \Delta \nu}=\left[\begin{array}{cc}
2.7062 & -0.0914 \\
-0.0914 & 0.0188
\end{array}\right]
$$

Here, the DEM error (first parameter) is in meters, and the deformation rate (the second parameter) is in millimeters/year. It is shown therefore from (23) that the standard deviations of the estimated parameters are $1.64 \mathrm{~m}$ and $0.137 \mathrm{~mm} / \mathrm{year}$, respectively.

Once the double-difference parameters at the arcs are determined, the parameters at the points can be obtained by spatial integration (when one reference point is assumed). Fig. 7 shows the errors in the estimated DEM and the deformation rates at the TCP, i.e., difference between the estimated and the true values. A statistics of the errors is given in Table I. It can be seen that the estimated DEM accuracy is not as high as that typically estimated with PSInSAR. This is mainly due to the fact that only interferograms with short baselines are used in the solution.

\section{B. Estimation of Nonlinear Deformation Signal}

A nonlinear deformation signal is simulated to assess the performance of the proposed method in areas experiencing complex deformation. The model for ground deformation in the LOS direction takes the following form:

$$
d(T)=-15 T+3 T^{2}+0.2 T^{3} .
$$

TABLE II

STATISTICS OF ERRORS IN THE ESTIMATEd COEFFICIENTS

\begin{tabular}{ccccc}
\hline Coefficient & $\min$ & $\max$ & mean & Std* \\
\hline Linear term $(\mathrm{mm} / \mathrm{y})$ & -6.58 & 2.63 & -1.94 & $1.43(1.25)$ \\
Quadratic term $\left(\mathrm{mm} / \mathrm{y}^{2}\right)$ & -0.91 & 1.46 & 0.35 & $0.35(0.31)$ \\
Cubic term $\left(\mathrm{mm} / \mathrm{y}^{3}\right)$ & -0.09 & 0.07 & -0.017 & $0.024(0.021)$ \\
\hline
\end{tabular}

(*): Standard deviation from Eq.(14)

The coefficients to be estimated at the TCP are shown in Fig. 8(a)-(c). By updating the design matrix, i.e., $A$ in (7), the least squares model can be used directly for nonlinear parameter estimation. The errors in the estimated coefficients (compared with the simulated input) are shown in Fig. 8(e) and (f). A statistics of the errors at the TCP is given in Table II. It can be seen from the results that the proposed method works well with the nonlinear deformation signal, although the estimation accuracy is not as high as in the case of the linear signals. This is mainly due to the limited number of observations available, i.e., interferograms. In order to get more accurate estimation of complex deformation signals, more interferograms should be used.

\section{Comparison With Unweighted Least Squares Solution}

A least squares solution is derived without using the weight matrix to assess the impact of applying the weight matrix. It is found from the results obtained that the standard deviation of the estimated deformation rates is degraded from 0.16 to $0.41 \mathrm{~mm} /$ year (Table III) when no weight matrix is used. The results also become worse for the case of nonlinear deformations. This indicates that a proper weighting scheme is important and should be used for modeling multiple SAR acquisitions. 
TABLE III

COMPARISON BETWEEN ERRORS IN THE ESTIMATED DEFORMATION RATE USING WEIGHT AND NONWEIGHT MODELS

\begin{tabular}{cccccc}
\hline & & $\min$ & $\max$ & $\operatorname{mean}$ & std \\
\hline \multirow{2}{*}{$\begin{array}{c}\text { Linear defo. rate } \\
(\mathrm{mm} / \mathrm{y})\end{array}$} & $\begin{array}{c}\text { with } \\
\text { weight }\end{array}$ & -0.45 & -0.41 & -0.008 & 0.164 \\
\cline { 2 - 6 } & $\begin{array}{l}\text { without } \\
\text { weight }\end{array}$ & -1.14 & 1.49 & 0.037 & 0.41 \\
\hline
\end{tabular}

\section{Computational Burden}

The whole processing takes about $1.5 \mathrm{~min}$ for the aforementioned simulated data. Since the selected area and the number of TCPs in the simulated test are relatively small, it is difficult to evaluate the efficiency of the proposed method. We address here the issue of computational burden with figures from a case of an expanded area of $70 \mathrm{~km}$ by $50 \mathrm{~km}$. In this case, 201778 TCP points are selected, and a grid with $150-\mathrm{m}$ spacing is placed over the area. All the points within a radius of $250 \mathrm{~m}$ centered at the grid node are connected. This task takes about $5 \mathrm{~h}$ using a computer with Intel core2 duo CPU (T9600 at $2.8 \mathrm{GHz}$ ) and 4-GB memory. Some 1176922 arcs (each arc has 55 observations) are then formed. The least squares solution only takes less than $3 \mathrm{~min}$.

\section{CONCLUSION}

A least-squares-based method for deformation estimation using multitemporal SAR data has been developed and validated with simulated data. The method includes new approaches for forming local Delaunay triangulation network, detecting and removing arcs with phase ambiguities, and properly weighting of phase observations, and a least squares model for obtaining the solutions. A distinctive advantage of the method is that it does not require phase unwrapping that is usually time consuming and often error prone. The results from controlled tests have shown that the method works well in areas with either linear or nonlinear ground deformations. The study has also shown that proper weighting of the phase observations is very important to achieve the optimal solutions.

Although the proposed method can allow a complicated deformation model to be used, caution should be exercised in choosing the model to avoid overparameterization. It should also be noted that only the a priori $\mathrm{VC}$ matrix is used in the method to calculate the weight matrix for the least squares model. More investigations should be made to study to further improve the determination of the weight matrix.

\section{ACKNOWLEDGMENT}

The authors would like to thank Prof. R. Hanssen and Dr. B. Kampes for sharing the InSAR simulation package [27], Dr. B. Kampes for releasing the demo codes of STUN algorithm with [13], and the anonymous reviewers for their valuable comments and suggestions.

\section{REFERENCES}

[1] H. A. Zebker and J. Villasenor, "Decorrelation in interferometric radar echoes," IEEE Trans. Geosci. Remote Sens., vol. 30, no. 5, pp. 950-959, Sep. 1992.

[2] Z. Lu, O. Kwoun, and R. Rykhus, "Interferometric synthetic aperture radar (InSAR): Its past, present and future," Photogramm. Eng. Remote Sens., vol. 73, no. 3, pp. 217-221, Mar. 2007.

[3] A. Ferretti, C. Prati, F. Rocca, and D. di Elettronica, "Nonlinear subsidence rate estimation using permanent scatterers in differential SAR interferometry," IEEE Trans. Geosci. Remote Sens., vol. 38, no. 5, pp. 2202-2212, Sep. 2000.

[4] C. Colesanti, A. Ferretti, F. Novali, C. Prati, and F. Rocca, "SAR monitoring of progressive and seasonal ground deformation using the permanent scatterers technique," IEEE Trans. Geosci. Remote Sens., vol. 41, no. 7, pp. 1685-1701, Jul. 2003.

[5] A. Refice, F. Bovenga, and R. A. Nutricato, "MST-based stepwise connection strategies for multipass radar data, with application to coregistration and equalization," IEEE Trans. Geosci. Remote Sens., vol. 44, no. 8, pp. 2029-2040, Aug. 2006.

[6] B. Kampes and R. Hanssen, "Ambiguity resolution for permanent scatterer interferometry," IEEE Trans. Geosci. Remote Sens., vol. 42, no. 11, pp. 2446-2453, Nov. 2004.

[7] A. Hooper, H. Zebker, P. Segall, and B. Kampes, "A new method for measuring deformation on volcanoes and other natural terrains using InSAR persistent scatterers," Geophys. Res. Lett., vol. 31, pp. 611-615, Dec. 2004.

[8] C. Werner, U. Wegmuller, A. Wiesmann, T. Strozzi, G. Sensing, and S. Muri, "Interferometric point target analysis with JERS-1 L-band SAR data," in Proc. IGARSS, Toulouse, France, Jul. 21-25, 2003, pp. 4359-4361.

[9] P. Berardino, G. Fornaro, R. Lanari, and E. Sansosti, "A new algorithm for surface deformation monitoring based on small baseline differential SAR interferograms," IEEE Trans. Geosci. Remote Sens., vol. 40, no. 11, pp. 2375-2383, Nov. 2002.

[10] R. Lanari, O. Mora, M. Manunta, J. Mallorqui, P. Berardino, and E. Sansosti, "A small-baseline approach for investigating deformations on full-resolution differential SAR interferograms," IEEE Trans. Geosci. Remote Sens., vol. 42, no. 7, pp. 1377-1386, Jul. 2004.

[11] P. Tizzani, P. Berardino, F. Casu, P. Euillades, M. Manzo, G. P. Ricciardi, G. Zeni, and R. Lanari, "Surface deformation of Long Valley Caldera and Mono Basin, California, investigated with the SBAS-InSAR approach," Remote Sens. Environ., vol. 108, no. 3, pp. 277-289, Jun. 2007.

[12] S. Usai, "A least-squares database approach for SAR interferometric data," IEEE Trans. Geosci. Remote Sens., vol. 41, no. 4, pp. 753-760, Apr. 2003.

[13] B. Kampes, Radar Interferometry: Persistent Scatterer Technique. Dordrecht, The Netherlands: Springer-Verlag, 2006.

[14] P. J. G. Teunissen, Adjustment Theory: An Introduction, 1st ed. Delft, The Netherlands: Delft Univ. Press, 2000.

[15] K. Koch, Parameter Estimation and Hypothesis Testing in Linear Models, 2nd ed. Berlin, Germany: Springer-Verlag, 1999.

[16] A. Ferretti, G. Savio, R. Barzaghi, A. Borghi, S. Musazzi, F. Novali, C. Prati, and F. Rocca, "Submillimeter accuracy of InSAR time series: Experimental validation," IEEE Trans. Geosci. Remote Sens., vol. 45, no. 5, pp. 1142-1153, May 2007.

[17] F. Casu, M. Manzo, A. Pepe, and R. Lanari, "SBAS-DInSAR analysis of very extended areas: First results on a $60000-\mathrm{km}^{2}$ test site," IEEE Geosci. Remote Sens. Lett., vol. 5, no. 3, pp. 438-442, Jul. 2008.

[18] A. Ferretti, C. Prati, and F. Rocca, "Permanent scatterers in SAR interferometry," IEEE Trans. Geosci. Remote Sens., vol. 39, no. 1, pp. 8-20, Jan. 2001.

[19] P. Shanker and H. Zebker, "Persistent scatterer selection using maximum likelihood estimation," Geophys. Res. Lett., vol. 34, no. 22, p. 4, Nov. 2007.

[20] C. Rao and S. Mitra, Generalized Inverse of a Matrix and Its Applications. New York: Wiley, 1971

[21] P. Z. Jia and Z. Z. Tao, Optimal Estimation and Its Applications. Beijing, China: Science Press, 1984.

[22] R. Hanssen, Radar Interferometry: Data Interpretation and Error Analysis. Dordrecht, The Netherlands: Kluwer, 2001.

[23] S. Williams, Y. Bock, and P. Fang, "Integrated satellite interferometry: Tropospheric noise, GPS estimates and implications for interferometric synthetic aperture radar products," J. Geophys. Res.-Solid Earth, vol. 103, no. B11, pp. 27 051-27 067, 1998.

[24] Z. W. Li, X. L. Ding, and G. X. Liu, "Modeling atmospheric effects on InSAR with meteorological and continuous GPS observations: Algorithms 
and some test results," J. Atmos. Sol. Terr. Phys., vol. 66, no. 11, pp. 907917, Jul. 2004

[25] Z. W. Li, X. L. Ding, C. Huang, G. Wadge, and D. W. Zheng, "Modeling of atmospheric effects on InSAR measurements by incorporating terrain elevation information," J. Atmos. Sol. Terr. Phys., vol. 68, no. 11, pp. 1189-1194, Jul. 2006.

[26] G. X. Liu, S. M. Buckley, X. L. Ding, Q. Chen, and X. J. Luo, "Estimating spatiotemporal ground deformation with improved permanent-scatterer radar interferometry," IEEE Trans. Geosci. Remote Sens., vol. 47, no. 8, pp. 2762-2772, Aug. 2009.

[27] R. Hanssen and B. Kampes, InSAR Matlab Toolbox, Aug. 2005. [Online]. Available: http://enterprise.lr.tudelft.nl/doris/

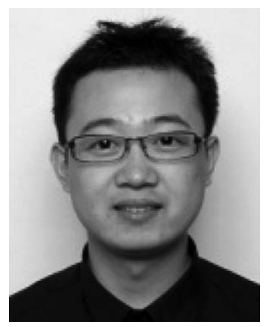

Lei Zhang ( $\mathrm{S}^{\prime} 08$ ) was born in Yantai, China, in 1981. He received the M.Sc. degree in geodesy and geodynamics from Tongii University, Shanghai, China, in 2007, with a thesis on fault slip inversion with InSAR and GPS data based on a triangular dislocation model. He is currently working toward the Ph.D. degree in the Department of Land Surveying and Geo-Informatics (LSGI), The Hong Kong Polytechnic University, Kowloon, Hong Kong.

His research interests focus on developing advanced processing techniques for multiple SAR images and the application of multitemporal interferometric analysis to the retrieval of geophysical parameters and displacement monitoring, with emphasis on natural hazards.

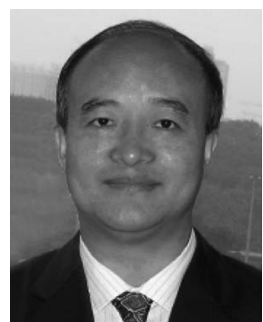

Xiaoli Ding received the B.Eng. degree from the Central South University of Metallurgy, Changsha, China, in 1983 and the Ph.D. degree from the University of Sydney, Sydney, N.S.W., Australia, in 1993.

$\mathrm{He}$ is currently the Chair Professor of geomatics and the Head of the Department of Land Surveying and Geo-Informatics, The Hong Kong Polytechnic University, Kowloon, Hong Kong. He has lectured at the Northeast University of Technology, Shenyang, China (in 1983-1986) and the Curtin University of Technology, Perth, W.A., Australia (in 1992-1996), before joining The Hong Kong Polytechnic University in 1996. His main research interests are in developing technologies for studying ground and structural deformation and geohazards, with a current focus being upon spaceborne geodetic technologies such as GPS and InSAR.

Prof. Ding is the President of Sub-Commission 4.4 of the International Association of Geodesy (IAG) on airborne and spaceborne imaging technologies. $\mathrm{He}$ is also a Fellow of the IAG.

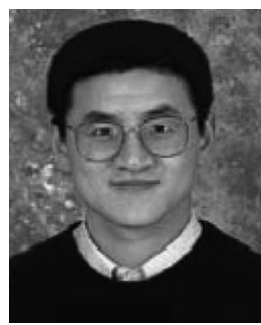

Zhong Lu (S'96-A'97-M'97-SM'07) received the M.S. degree from Beijing University, Beijing, China, in 1992 and the Ph.D. degree from the University of Alaska Fairbanks, Fairbanks, in 1996.

$\mathrm{He}$ is currently with the Earth Resources Observation and Science Center and the Cascades Volcano Observatory, U.S. Geological Survey (USGS), Vancouver, WA, as a Physical Scientist. He is the Principal Investigator of projects funded by NASA, the European Space Agency, Japan Aerospace Exploration Agency, German Space Agency, and USGS on the study of land surface deformation using satellite InSAR imagery. His research interests include technique developments of SAR, InSAR, and persistent scatterer InSAR processing and their applications on natural hazard monitoring and natural resources management.

Dr. Lu has been a committee member of the International User Team for Radarsat-C SAR Constellations, the GeoEarthscope InSAR User Working Group, and NASA's Alaska Satellite Facility User Working Group. He serves on the editorial boards of three international journals. He was the recipient of the 2001 Raytheon Distinguished Level Award for Excellence in Technology and the 1999 Jerald J. Cook Memorial Award. 